\title{
Next-generation sequencing and targeted cancer therapy
}

\section{Editorial}

The complex nature of cancer and the transformation of cancer care, prompted by advanced understanding of genomic subtypes and emergence of targeted therapies, make the detection of genetic alterations to guide therapeutic decision-making more critical than ever. The primary goal of targeted therapies is to fight tumor cells with more precision and potentially fewer side effects. Today, the success of precision cancer therapy is heavily replied on state-ofthe-arttechnologies to identify actionable genetic alterations and monitordynamic clonal evolution during tumor progression to help determine appropriate therapies for cancer patients. ${ }^{1}$ Because not every cancer develops in the same way in each person, targeted therapy is personalized to the individual. Targeted therapy at molecule level potentiallyis less harm to normal cells, thus has fewer side effects, with improved efficacy and quality of life. A number of targeted therapies have been implemented into standard of care, such as the selective BRAF inhibitor vemurafenib, Bcr-Abll drugs imatinib and nilotinib, EGFR antagonist serlotinib and gefitinib, HER2 therapeutic antibody trastuzumab, and ALK inhibitor crizotinib. In these cases, genetic biomarkers as companion diagnostics able to stratify patient responders have been crucial to the successful outcome of targeted therapies. ${ }^{2,3}$ As we understand more and more about cancer biology, we are now able to develop drugs that selectively target the molecular drivers of tumorigenesis. Cancer genomes are extremely heterogeneous and most probably would have to be scanned multiple times to monitor disease progression, as tumor cells are constantly evolving and developing resistance to anti-cancer drugs. Nextgeneration sequencing (NGS) technologies, in this respect, have the ability to massively parallelize the sequencing of millions of DNA templates, simultaneously interrogate thousands of clinicallyactionable mutations, revolutionizing the way in which we detect, treat and manage cancer patients. ${ }^{4-7}$

Over the past few years, the data derived from NGS provided tremendous insights into the pathophysiology of cancer genomics, simultaneously providing new diagnostic and therapeutic information, as well as identifying specific lesions for targeted therapy. The advantages of NGS in genome medicine are many, including surveillance of the whole genome for the detection of all types of mutations. Major barriers for clinical implementation include high cost of equipment and reagents, the requirement for relatively large batch sizes for each run, large data sets, the expense complexity of analysis and interpretation, and the findings with unknown clinical significance. However, we have already observed an enormous decrease in cost during the past few years with the same level of information being delivered faster. Further, focused gene panels for targeted cancer sequencing significantly narrow the scope of a sequencing project, reducing cost and data analysis burdens. Because it assesses a limited set of genes, targeted cancer sequencing allows for deeper coverage of those genes and higher sensitivity to call variants in rare tumor subclones confidently. In this aspect, targeted
Volume 2 Issue 3 - 2015

\author{
Chen-Hsiung Yeh \\ CirculoGene Diagnostics, USA
}

Correspondence: Chen-Hsiung Yeh, CirculoGene Diagnostics, USA,Tel I2052340I28, Email cyeh@circulogene.com

Received: July 23, 2015 | Published: July 27, 2015

gene sequencinghas become a preferable testing platform which is more cost effective, the results are easy to interpret, and most findings are clinically actionable for cancer-relevant genes. NGS panels for cancer-associated genes known in leukemia, as well as lung, colon, breast, melanoma and other cancers are currently provided by some commercial laboratories.

Since its introduction in 2007, NGS technology has already made extraordinary advances, producing data on an unprecedented scale, this technique is now driving the generation of knowledge (especially in oncology) to new dimensions. ${ }^{8,9}$ It's not feasible for every cancer patient to receive whole genome sequencing and analysis, but it's clear that there's already a place for it in the medical toolbox. While the task of deciphering the cancer genome remains ongoing, we are already beginning to see clinical application of NGS-based genetic testing. And as NGS becomes faster and less expensive, it's sure to be used more frequently and with greater benefit. Most importantly, the convergence of NGS technologies, bioinformatics power and algorithms, and other genomics advancements brings precision medicine closer than ever to a cost- and time-effective reality, and along with it the promise of enormous improvements in drug discovery, diagnostics, and personalized treatment of patients. To put the power of NGS into perspective, the Human Genome Project, based on Sanger sequencing, required 5years and $\$ 300$ million to sequence a single genome. Today, the same task can be achieved by NGS in a matter of weeks for a mere $\$ 5000^{10}$ (Table 1). It will be only a matter of time before the long sought-after goal of the $\$ 1000$ genome set by the U.S. National Institutes of Health will be achieved. NGS innovation allows clinicians to make the best possible therapeutic choices, minimize the use of ineffective therapies and enhance enrollment in clinical trials appropriate for the individual patient. As personalized medicine, targeted therapies and treatments become the norm, the future of NGS-based genetic testing's role in cancer patient care is expanding. And the role of the clinical laboratory is becoming more prominent as a resource for understanding each patient individually at the genetic level. Cost, turnaround time, and interpretability of NGS data will evolve and improve rapidly, as in many other technology sectors. We are at the dawn of an incredible precision medicine era. 
Table I Trends in next-generation sequencing

\begin{tabular}{lll}
\hline Key characteristics & Sanger sequencing & Next-generation sequencing \\
\hline Fundamental Technology & Sequencing by Termination & Sequencing by Synthesis \\
Resolution/Sensitivity & Low & High \\
Raw read Accuracy & Fair & High \\
Read Length & $50-250 \mathrm{bp}$ & $<400 \mathrm{bp}$ \\
Throughput & Very Low (single gene) & Very High (multiple genes) \\
Cost & Low & Fair \\
Start-to-Finish & 2-3days/run & $5-7$ days/run \\
Sample Preparation & Less Complex & Complex \\
Data Analysis & Simple & Complex \\
\hline
\end{tabular}

\section{Acknowledgements}

None.

\section{Conflict of interest}

The author declares no conflict of interest.

\section{References}

1. Brooks JD. Translational genomics: The challenge of developing cancer biomarkers. Genome Res. 2012;22(2):183-187.

2. Weinstein IB. Addiction to oncogenes-the Achilles heal of cancer. Science. 2002;297(5578):63-64.

3. Mills GB. An emerging toolkit for targeted cancer therapies. Genome Res. 2012;22(2):177-182.

4. Harris TJ, McCormick F. The molecular pathology of cancer. Nat Rev Clin Oncol. 2010;7(5):251-265.
5. Macconaill LE, Garraway LA. Clinical implications of the cancer genome. J Clin Oncol. 2010;28(35):5219-5228.

6. Chin L, Hahn WC, Getz G, et al. Making sense of cancer genomic data. Genes Dev. 2011;25(6):534-555.

7. Roychowdhury S, Iyer MK, Robinson DR, et al Personalized oncology through integrative high-throughput sequencing: a pilot study. Sci Transl Med. 2011;3(111):111ra121.

8. Sheun A, Foulkes WD. Clinical implications of next generation sequencing for cancer medicine. Curr Oncol. 2010;17(5):39-42.

9. Hui P. Next generation sequencing: Chemistry, technology and applications. Top Curr Chem. 2014;336:1-18.

10. Drmanac R, Sparks AB, Callow MJ, et al. Human genome sequencing using unchained base reads on self-assembling DNA nanoarray. Science. 2010;327(5961):78-81 